\title{
A Randomized Controlled Trial of Continuous Labor Support for Middle-Class Couples: Effect on Cesarean Delivery Rates
}

\author{
Susan K. McGrath, PhD, and John H. Kennell, MD
}

\begin{abstract}
Background: Previous randomized controlled studies in several different settings demonstrated the positive effects of continuous labor support by an experienced woman (doula) for low-income women laboring without the support of family members. The objective of this randomized controlled trial was to examine the perinatal effects of doula support for nulliparous middle-income women accompanied by a male partner during labor and delivery. Methods: Nulliparous women in the third trimester of an uncomplicated pregnancy were enrolled at childbirth education classes in Cleveland, Ohio, from 1988 through 1992. Of the 686 prenatal women recruited, 420 met enrollment criteria and completed the intervention. For the 224 women randomly assigned to the experimental group, a doula arrived shortly after hospital admission and remained throughout labor and delivery. Doula support included close physical proximity, touch, and eye contact with the laboring woman, and teaching, reassurance, and encouragement of the woman and her male partner. Results: The doula group had a significantly lower cesarean delivery rate than the control group $(13.4 \% \mathrm{vs} 25.0 \%$, $\mathrm{p}=0.002)$, and fewer women in the doula group received epidural analgesia $(64.7 \% \mathrm{vs} 76.0 \%, \mathrm{p}=$ 0.008). Among women with induced labor, those supported by a doula had a lower rate of cesarean delivery than those in the control group $(12.5 \%$ vs $58.8 \%, \mathrm{p}=0.007)$. On questionnaires the day after delivery, 100 percent of couples with doula support rated their experience with the doula positively. Conclusions: For middle-class women laboring with the support of their male partner, the continuous presence of a doula during labor significantly decreased the likelihood of cesarean delivery and reduced the need for epidural analgesia. Women and their male partners were unequivocal in their positive opinions about laboring with the support of a doula. (BIRTH 35:2 June 2008)
\end{abstract}

Key words: doula, labor support, cesarean delivery, epidural analgesia

Susan K. McGrath is an Adjunct Faculty and John H. Kennell is a Professor Emeritus in the Department of Pediatrics, Case Western Reserve University, Cleveland, Ohio, USA.

Address correspondence to Susan K. McGrath, PhD, Department of Pediatrics, Case Western Reserve University, Rainbow Babies and Children's Hospital, 11100 Euclid Avenue, MS 6038, Cleveland, OH 44106, USA.

Accepted November 21, 2007

This research was supported in full by Grant HD 16915 awarded by the National Institute of Child Health and Human Development, Bethesda, Maryland, USA.

(C) 2008, Copyright the Authors

Journal compilation () 2008, Wiley Periodicals, Inc.
Previous randomized controlled studies in several different settings demonstrated the positive effects of continuous labor support by an experienced woman (doula) for low-income women laboring without the support of family members (1-7). "Doula" is a Greek word that describes an experienced woman who aids and supports a new mother with infant care tasks. In this study, the term doula refers to a woman who provides continuous labor support.

Two studies in Guatemala showed a significant reduction in the length of labor, in the rate of cesarean deliveries, in the rate of perinatal problems, and in the need for medications in the groups who received doula 
support $(1,2)$. A study involving 616 nulliparous lowincome women delivering at a technologically sophisticated Houston hospital compared women who received continuous doula support with a control group who received routine obstetric care (3). The women supported by a doula during labor required fewer cesarean or forceps deliveries and less epidural analgesia, had shorter labors, needed less oxytocin augmentation, and were less likely to develop fever during labor. In a similar study of 189 nulliparous women in South Africa, Hofmeyr et al reported that participants in the control group required obstetric analgesia significantly earlier after enrollment than did women who were supported by a doula (4). A Canadian study demonstrated that laboring women who received continuous labor support required less anesthesia or analgesia than did women in a control group (5). A meta-analysis of these five studies and a sixth randomized controlled trial from Finland (6) demonstrated that doula support resulted in a 50 percent reduction in cesarean deliveries, a 30 percent decrease in the use of pain medication, and a 60 percent decrease in requests for epidural analgesia (7).

Although these results provide evidence about the benefits of doula support, questions remained about the generalizability of the positive effects for middle and upper income groups or for women accompanied by their male partners or other family members during labor and delivery. Because fathers provide support to most laboring women in United States hospitals, the presence of a doula is often thought to be unnecessary. Furthermore, middle-class couples can attend a hospitalor community-based childbirth education class and have access to print and online publications that can prepare them for the new experience of labor and delivery.

The effectiveness of doula support in reducing cesarean deliveries and improving perinatal outcome for low-income childbearing women who are not supported by family members during labor is well documented. The present randomized controlled trial was designed to examine the effects of doula support for middle and upper income nulliparous women who were also accompanied by a male partner throughout labor and delivery.

\section{Methods}

\section{Prenatal Procedures: Recruitment and Consent}

Nulliparous women between the ages of 18 and 41 years in the third trimester of an uncomplicated pregnancy who expected to be accompanied during labor by their male partner and planned to deliver at University Hospitals in Cleveland, Ohio, USA, under the care of a private obstetrician were asked to participate in the study. The project was approved by the hospital's Investigative Review Board for quality of design and ethical integrity and was reviewed annually. Women were enrolled at childbirth education classes in the greater Cleveland area from October 1988 through October 1992.

After signing the consent form, women and their male partners were given a packet of questionnaires that were to be completed and mailed back to the study office. Hospital charts were flagged, and both labor and delivery personnel and study participants were asked to telephone the study research coordinator when an enrolled couple arrived at the hospital to deliver their baby. A total of 686 expectant mothers volunteered for this study. Of these women, $168 \mathrm{did}$ not meet enrollment criteria, 79 were excluded because study personnel were not notified when these women arrived at the hospital in labor, and an additional 19 were excluded from the study because they chose a midwife delivery.

A final total of 420 women (388 accompanied by their male partner, 29 accompanied by some other support person, and 3 who were alone) were randomly assigned to either the doula $(n=224)$ or the control $(n=196)$ group. The target sample size for this research was 200 women in each group. This number was based on a power analysis using the then-current cesarean delivery rate at the study hospital of 24 percent, with a 50 percent projected reduction in the experimental group, an alpha level of 0.05 , and a power of 0.80 .

\section{Procedures During Labor and Delivery: Random Assignment and Intervention}

When the research coordinator was informed that an enrolled woman had arrived at the hospital in early active labor, the coordinator opened the next sequentially numbered opaque envelope to determine random assignment to the doula or control group. Couples assigned to the control group received routine obstetric and nursing care. For couples in the doula group, a doula met them at the hospital as soon as possible after random assignment (typically within an hour of their arrival at the hospital) and remained with them throughout labor and delivery. The central component of doula support was the doula's continuous bedside presence during labor and delivery, although her specific activities were individualized to the needs of the laboring couple. In general, the doulas used verbal encouragement and reassurance, teaching, touch, eye contact, and close physical proximity to support the laboring woman and her partner as a unit. The doulas guided the partner to support the 
laboring woman and were careful not to take over the partner's role.

Over the 4-year span of the study, 10 women served as research doulas, with 3 of these women attending 79 percent of the doula-supported labors. The doulas ranged in age from 24 to 44 years, with an average of 15 years of education. One had never married, one was African American, and most of them had experience as childbirth educators. They all completed training requirements that were equivalent to Doulas of North America International (DONA International) doula certification. All the doulas worked in this capacity on a part-time, on-call basis and were paid a set amount from the research grant for each labor they attended.

\section{Procedures after Delivery: Data Collection}

After delivery and before hospital discharge, demographic information was collected from participating couples. Research assistants had access to both the mother's and the newborn's hospital charts to gather detailed information about procedures and interventions during labor and delivery. Couples were asked to complete a packet of questionnaires about their labor and delivery experience. Completed questionnaires were left with the research assistant before hospital discharge. Approximately 6 weeks after delivery, participating couples were sent a final packet of questionnaires. When these were returned by mail to the study office, couples received a payment of $\$ 25$ for their participation.

\section{Data Analysis}

Descriptive statistics including frequencies, percentages, means, and standard deviations were used to summarize the demographic characteristics of the study sample. Statistical analyses were performed as two-way comparisons of the doula and control groups. A chi-square test was used to analyze discrete variables; continuous variables were analyzed using one-way analysis of variance. Statistical analyses were done with SPSS version 9.0.1 (8).

\section{Results}

All the 420 women who participated in this study were classified as middle or upper income and were delivered by private obstetricians. Seventy-eight percent of participants were white; 88 percent were married. All but two of the women were high school graduates, with 57 percent of them also having a college degree.

Table 1. Demographic Data

\begin{tabular}{|c|c|c|c|}
\hline Variable & Doula Group $(\mathrm{n}=224)$ & Control Group $(\mathrm{n}=196)$ & $\mathrm{p}$ \\
\hline Maternal age (yr) & $\overline{\mathrm{x}}=28.97(\mathrm{SD}=4.83)$ & $\overline{\mathrm{x}}=28.60(\mathrm{SD}=4.49)$ & 0.42 \\
\hline Maternal race & & & 0.47 \\
\hline Caucasian & $180(80.4 \%)$ & $149(76.0 \%)$ & \\
\hline African American & $37(16.5 \%)$ & $43(21.9 \%)$ & \\
\hline Asian & $6(2.7 \%)$ & $3(1.5 \%)$ & \\
\hline Hispanic & $1(0.4 \%)$ & $1(0.5 \%)$ & \\
\hline Maternal education & & & 0.16 \\
\hline$<$ High school & $2(0.9 \%)$ & $0(0 \%)$ & \\
\hline High school graduate & $19(8.5 \%)$ & $23(11.7 \%)$ & \\
\hline Some college & $57(25.4 \%)$ & $43(21.9 \%)$ & \\
\hline College graduate or more & $132(59.0 \%)$ & $109(55.6 \%)$ & \\
\hline Unknown & $14(6.3 \%)$ & $21(10.7 \%)$ & \\
\hline Marital status & & & 0.62 \\
\hline Single & $18(8.0 \%)$ & $21(10.7 \%)$ & \\
\hline Married & $200(89.3 \%)$ & $169(86.2 \%)$ & \\
\hline Cohabiting & $6(2.7 \%)$ & $6(3.1 \%)$ & \\
\hline Age at first prenatal visit (wk) & $\overline{\mathrm{x}}=10.2(\mathrm{SD}=4.20)$ & $\overline{\mathrm{x}}=9.7(\mathrm{SD}=4.31)$ & 0.36 \\
\hline \multicolumn{4}{|l|}{ Labor support person } \\
\hline Male partner/baby's father & $205(91.5 \%)$ & $183(93.4 \%)$ & 0.48 \\
\hline Mother's mother & $7(3.1 \%)$ & $8(4.1 \%)$ & \\
\hline Other female family member & $6(2.7 \%)$ & $1(0.5 \%)$ & \\
\hline Female friend & $4(1.8 \%)$ & $3(1.5 \%)$ & \\
\hline None & $2(0.9 \%)$ & $1(0.5 \%)$ & \\
\hline Gestational age at birth (wk) & $\overline{\mathrm{x}}=39.8(\mathrm{SD}=1.34)$ & $\overline{\mathrm{x}}=40.0(\mathrm{SD}=1.16)$ & 0.20 \\
\hline Birthweight (g) & $\overline{\mathrm{x}}=3456(\mathrm{SD}=457.3)$ & $\overline{\mathrm{x}}=3451(\mathrm{SD}=493.1)$ & 0.91 \\
\hline 5 -min Apgar $\leq 7$ & $4(1.8 \%)$ & $6(3.1 \%)$ & 0.30 \\
\hline
\end{tabular}


The average maternal age was 28 years. The doula and control groups were similar in race, marital status, maternal education, and maternal age (Table 1).

Although the study was originally planned to include only those women supported by their male partners during labor, the 32 enrolled participants who arrived at the hospital without their male partner were included in the analysis. Thus, 92 percent of women were supported by their male partner and 7 percent by a female family member or friend. Three women who labored without a family support person accounted for the remaining 1 percent.

\section{Obstetric Events}

To describe the obstetric environment at the time of this study, all 420 participants were analyzed together regardless of group assignment. Seventy percent $(n=$ 294) of the participants received epidural analgesia during labor, and 57 percent $(n=240)$ were given oxytocin to augment labor. Oxytocin was started before the onset of labor to induce labor in 33 of these women. The mean labor length for all study women was 13.6 hours $(\mathrm{SD}=6.5 \mathrm{hr})$. Twenty-one percent of the 420 deliveries $(n=90)$ required forceps or vacuum extraction and 19 percent $(n=79)$ were by cesarean section. During the course of labor, 17 percent of the women $(n=73)$ developed a temperature of $37.5^{\circ} \mathrm{C}$, with 7 percent $(n=29)$ reaching $38.0^{\circ} \mathrm{C}$ or greater.

The cesarean delivery rate for women in the doula group $(13.4 \%)$ was significantly lower than the rate for women in the control group $(25.0 \% ; p=0.002)$ (Table 2). When only the 388 women supported during labor by their male partner were considered, the presence of the doula had a significant effect on the cesarean delivery rate, 13.7 percent in the doula group compared with 25.7 percent in the control group ( $p=$ 0.002 ). The cesarean delivery rate among women in the control group who had induced labor was 58.8 percent (10 of 17 women). Those who had induced labor and were supported by a doula had a significantly lower rate of 12.5 percent ( 2 of 16 women, $p=0.007$ ) (Table 2).

Fewer women in the doula group required epidural analgesia $(64.7 \%)$ when compared with women in the

Table 2. Outcomes

\begin{tabular}{lrrr}
\hline & $\begin{array}{c}\text { Doula Group } \\
\text { No. }(\%)\end{array}$ & $\begin{array}{c}\text { Control Group } \\
\text { No. (\%) }\end{array}$ & $\mathrm{p}$ \\
\hline Cesarean delivery & & & \\
$\quad$ All women & $30 / 224(13.4)$ & $49 / 196(25.0)$ & 0.002 \\
$\quad$ Induced women & $2 / 16(12.5)$ & $10 / 17(58.8)$ & 0.007 \\
Epidural analgesia & $145 / 224(64.7)$ & $149 / 196(76.0)$ & 0.008 \\
\hline
\end{tabular}

control group $(76.0 \% ; p=0.008)$ (Table 2). When only those woman who were supported by a male partner were included in the analysis, significantly fewer women in the doula group required epidural analgesia compared with women in the control group $(63.9 \%$ vs $75.4 \%, p=0.009)$.

During labor, 73 women $(17.4 \%)$ developed an elevated temperature (defined as $37.5^{\circ} \mathrm{C}$ ). The temperature of 29 participants $(6.9 \%)$ reached at least $38.0^{\circ} \mathrm{C}$, a common definition for fever. The development of elevated temperature was unrelated to the presence or absence of doula support but was highly correlated with epidural analgesia $(p=0.005)$. Of the 29 women with a temperature of at least $38.0^{\circ} \mathrm{C}, 27$ had received epidural analgesia $(p=0.002)$.

\section{Responses to Questionnaires}

To evaluate women's subjective experience of laboring with doula support, the questionnaires administered at 24 hours and 6 weeks postpartum included an item on the presence of the doula. Each woman and male partner in the doula group were asked to rate the presence of the doula on a 4-point scale ranging from "very positive" to "very negative." The percentages of doula-supported participants who completed the questionnaires were as follows: mothers at 24 hours postpartum $=87.9$ percent, mothers at 6 weeks postpartum $=87.5$ percent, fathers at 24 hours postpartum $=80.8$ percent, and fathers at 6 weeks postpartum $=75.5$ percent. On the questionnaires at 24 hours postpartum, all women and their male partners who were supported by doulas rated the presence of the doula as "positive" (7\%) or "very positive" $(93 \%)$. Eighty-six percent of the doula-supported respondents to the 6-week postpartum questionnaires rated the presence of the doula as "very positive," with 13 percent giving a "positive" rating and less than 1 percent $(n=2)$ a "negative" rating. The only mother who gave a negative rating to the doula at 6 weeks postpartum had a long arduous labor that culminated in a cesarean delivery (and her partner rated the doula positively). The only father who rated the doula negatively at 6 weeks postpartum stated that he and his wife would have preferred to labor alone, although his wife gave the doula a positive rating.

\section{Discussion}

The results from this study emphasize the positive effects of doula support during labor for middle and upper income women who are also accompanied by their male partners or other family support. 
Providing a doula to middle and upper socioeconomic status couples resulted in a statistically and clinically significant decrease in the rate of cesarean delivery in the experimental group. Whereas previous studies have demonstrated the impact of doula support for lower socioeconomic status women who had no family members present, all the participants in the current study were middle class or higher and all but three $(1 \%)$ were accompanied by a family member (most often the father of the baby).

Currently in the United States, fathers-to-be are usually expected to accompany their partner through labor and delivery. Fathers want to be present at the birth of their babies, and mothers want them there as well, but perhaps fathers should not be expected to fulfill the role of primary labor companion. Results from this study demonstrated that the support provided by male partners does not have the same positive impact on perinatal outcome as does that of experienced doulas. Doulas come to a labor with a specific well-defined role, and their relationship with the laboring woman ends soon after the birth. In contrast, a father-to-be may attend his infants' birth for a variety of reasons, and his role is far from clear. Although some new fathers have attended childbirth education classes or been exposed to childbirth in the media, they typically have had little or no direct experience with labor and delivery.

Even in industrialized cultures, childbirth remains within the realm of women, with boys and men having little exposure to the details of childbirth. In addition, the sights, sounds, and smells of the labor suite can be particularly upsetting. Giving support to a laboring woman often requires extreme patience and a "let it happen" attitude. A husband made anxious by his wife's pain and distress may want to assume a more active role and thus be less effective as a supportive companion. His emotional connection to his partner and their infant may interfere with his ability to remain calm and objective. Several studies have demonstrated that fathers' supportive behavior during labor differed from that of doulas $(9,10)$ and from that of female relatives (11). The presence of the father-tobe during labor and delivery is extremely important because of his relationship with his partner and the newborn, but it is the doula who provides support that can have a dramatic impact on perinatal outcome.

The effect of a doula may result from both the emotional and the instrumental support she provides to the laboring woman. Women supported by a doula repeatedly refer to the importance of their doula's presence, commenting that they could not have "made it through labor without my doula." The continuous presence of a caring individual may help decrease the laboring woman's anxiety. In addition, the doula's effect may stem from her specific behaviors - such as encouraging statements and soothing massagewhich may relax the woman and allow labor to follow its natural course. The constant presence of the doula may also have an effect on the medical staff, which, in turn, influences the course of labor. For example, an obstetrician may delay making a decision for epidural analgesia if the laboring woman remains calm because she is closely supported by a doula. Finally, the doula may affect outcomes by acting as an advocate so that the woman's viewpoint and opinions can be heard and considered when medical decisions are made. The exact source(s) of the doula's effect on labor and delivery outcomes remains a rich topic for future investigation.

Styles of obstetric management in U.S. hospitals have undergone striking changes since the first study of doula support began in 1984. Some authors have suggested that the increased use of epidural analgesia has been a cause of the concomitant increase in cesarean deliveries $(12,13)$. Considering the 70 percent rate of epidural analgesia in the reported study and an even higher current rate, the base level of cesarean deliveries has risen well above a 7 to 8 percent incidence. Interestingly, the 212 doula-supported women in the first U.S. randomized controlled trial of continuous labor support had an epidural rate of 8 percent and an identical cesarean delivery rate of 8 percent.

Although epidural analgesia can be an effective form of pain relief, its use can result in unwelcome side effects (12-15). The longer epidural analgesia is in place during labor, the greater the likelihood that the laboring woman will develop an elevated temperature (13). In the current study, epidural analgesia was highly correlated with temperature elevation in the laboring women $(p=0.005)$. The point at which a laboring woman's temperature is defined as a fever varies from hospital to hospital and may also include additional considerations, such as the results of prenatal cultures for group B streptococcus. With the diagnosis of possible chorioamnionitis based on the symptom of maternal fever, a series of procedures for care of the baby may be initiated, including a sepsis workup, antibiotics administration, prolonged hospital stay, and separation of mother and baby. Thus, costly, invasive, and time-consuming tests and treatments may be undertaken because of an elevated temperature that is a result of epidural analgesia. A study of 45 women randomized to receive epidural analgesia and 39 women who received continuous doula support for pain relief during childbirth used postpartum interviews to demonstrate that doula support is an effective pain management technique that is less expensive and presents virtually no risks compared with epidural analgesia (16). 
The practice of obstetric medicine in the U.S. over the past 30 years has increasingly focused on the use of sophisticated technology and techniques to actively manage the low-risk labors and deliveries of healthy women. In the midst of this scientific emphasis, a lowcost and safe intervention in the form of continuous doula support has been shown to have a positive impact on the labor and deliveries of all first-time mothers regardless of socioeconomic status, prenatal education, and level of social support. With serious questions being raised about the routine use and potential side effects of some technologic obstetric interventions commonly used with healthy mothers, such as electronic fetal monitoring and epidural analgesia, the risk-free value of doula support can no longer be ignored.

\section{Conclusions}

For middle and upper class American women accompanied by their male partner or other family member, continuous doula support through labor significantly decreases cesarean delivery rates and reduces the need for epidural analgesia. Women and their male partners who receive the support of a doula rate the experience positively. Continuous labor support by a doula is a risk-free obstetric technique that could benefit all laboring women and should be made available in all maternity units.

\section{References}

1. Sosa R, Kennell J, Klaus M, et al. The effect of a supportive companion on perinatal problems, length of labor, and mother-infant interaction. N Engl J Med 1980;303(11): 597-600.
2. Klaus M, Kennell J, Robertson S, Sosa R. Effects of social support during parturition on maternal and infant morbidity. Br Med J 1986;293(6547):585-587.

3. Kennell J, Klaus M, McGrath S, et al. Continuous emotional support during labor in a U.S. hospital. JAMA 1991; 265(17):2197-2201.

4. Hofmeyr GJ, Nikodem VC, Wolman WL, et al. Companionship to modify the clinical birth environment: Effects of progress and perception of labour and breastfeeding. $\mathrm{Br} \mathrm{J} \mathrm{Obstet}$ Gynaecol 1991;98(8):756-764.

5. Hodnett ED, Osborn R. Effect of continuous intrapartum professional support on childbirth outcomes. Res Nurs Health 1989;12(5):289-297.

6. Hemminki E, Virta AL, Koponen P, et al. A trial of continuous human support during labor: Feasibility, interventions, and mothers' satisfaction. J Psychosom Obstet Gynaecol 1990;11:339-350.

7. Klaus MH, Kennell JH, Berkowitz G, Klaus P. Maternal assistance and support in labor: Father, nurse, midwife, or doula? Clin Consult Obstet Gynecol 1992;4:211-217.

8. SPSS Inc. Statistical Package for the Social Sciences. Version 9.0.1. Chicago, Illinois, SPSS Inc., 1999.

9. Bertsch T, Nagashima-Whalen L, Dykeman S, et al. Labor support by first-time fathers: Direct observations. J Psychosom Obstet Gynaecol 1990;11:251-260.

10. Lohn B, Kennell JH, McGrath S. Comparison of fathers and doulas as supportive labor companions. Ped Res 1992;31:12A.

11. Brooks AK, Kennell JH, McGrath SK. Supportive behaviors of men and women during labor. Ped Res 1995;31:13A.

12. Fusi L, Steer PJ, Maresh MJ, Beard RW. Maternal pyrexia associated with the use of epidural analgesia in labor. Lancet 1989;1(8649):1250-1252.

13. Lieberman E, Lang JM, Frigoletto F, Jr, et al. Epidural analgesia, intrapartum fever, and neonatal sepsis evaluation. Pediatrics 1997;99(3):415-419.

14. Thorp JA, Hu DH, Albin RM, et al. The effect of intrapartum epidural analgesia on nulliparous labor: A randomized, controlled, prospective trial. Am J Obstet Gynecol 1993;169(4):851-858.

15. Ramin SM, Gambling DR, Lucas MJ, et al. Randomized trial of epidural versus intravenous analgesia during labor. Obstet Gynecol 1995;86(5):783-789.

16. McGrath SK, Kennell JH, Varadarajulu V. Doula support: A risk-free alternative for pain relief during childbirth. Ped Res 1997;41:16A. 